\title{
EL TRABAJO DOCENTE DURANTE LA DICTADURA CÍVICO-MILITAR EN CHILE (1973-1990): UNA MIRADA DESDE LAS POLÍTICAS PÚBLICAS EDUCACIONALES
}

\author{
Felipe Andres Zurita Garrido ${ }^{1}$
}

\section{RESUMEN}

Se presentan resultados de una investigación doctoral que tuvo como objetivo analizar la Política Pública Educacional referida al trabajo docente construida por la Dictadura CívicoMilitar en Chile (1973-1990). Se trabajó con hipótesis que resaltaron la importancia que el régimen otorgó al profesorado mediante regulaciones jurídicas y acciones represivas. Se contó con aportaciones teóricas de A. Gramsci y E. P. Thompson, las que facilitaron el trabajo de interrogación de diferentes fuentes, privilegiando entre estas la legislación e informes de derechos humanos. Las conclusiones confirman las hipótesis planteadas y agregan elementos nuevos que invitan a revisitar las transformaciones realizadas en este periodo sobre la actividad del profesorado y el sistema educativo chileno en general.

Palabras clave: trabajo docente, Dictadura Cívico-Militar, Chile, Políticas Públicas Educacionales.

\footnotetext{
${ }^{1}$ Universidad Bernardo O'Higgins (UBO), Santiago, Chile.
} 


\section{O TRABALHO DOCENTE DURANTE A DITADURA CIVIL-MILITAR NO CHILE (1973-1990): UM OLHAR DESDE AS POLÍTICAS PÚBLICAS EDUCACIONAIS}

\section{RESUMO}

São apresentados os resultados de uma pesquisa de doutorado que teve como objetivo analisar a Política Pública Educacional referente ao trabalho docente construída pela Ditadura CívicoMilitar no Chile (1973-1990). Trabalhou-se com hipóteses que ressaltaram a importância que o regime concedeu aos professores por meio de normas legais e ações repressivas. Contou-se com contribuições teóricas de A. Gramsci e E. P. Thompson, as que facilitaram o trabalho de interrogação de diferentes fontes, privilegiando entre elas a legislação e os relatórios de direitos humanos. As conclusões confirmam as hipóteses propostas e agregam novos elementos que convidam a revisitar as transformações realizadas neste período sobre a atividade do corpo docente e do sistema educacional chileno em geral.

Palavras-chave: trabalho docente, Ditadura Cívico-Militar, Chile, Políticas Públicas Educacionais.

\section{TEACHING WORK DURING THE CHILEAN CIVIC- MILITARY DICTATORSHIP (1973-1990): AN OVERVIEW FROM PUBLIC EDUCATIONAL POLICIES}

\section{ABSTRACT}

This article presents the results of doctoral research whose objective was to analyse the Public Educational Policy built by the Chilean Civic-Military Dictatorship (1973-1990), an analysis carried out especially with regard to teaching work. We worked with hypotheses that highlighted the importance that the Regime granted to teachers through legal regulations and repressive actions. Theoretical contributions from A. Gramsci and E. P. Thompson were considered, which facilitated the research work from different sources, privileging among these the Chilean legislation and human rights reports. Conclusions confirm the hypotheses and add new elements that invite to revisit the transformations made in this period about the activity of teachers and the Chilean educational system, in general.

Keywords: teaching work, Chilean Civic-Military Dictatorship, Public Educational Policies.

\section{LE TRAVAIL D'ENSEIGNANT PENDANT LA \\ DICTATURE CIVILO-MILITAIRE AU CHILI (1973- 1990): UN REGARD DEPUIS LES POLITIQUES PUBLIQUES EN MATIĖRE D'ÉDUCATION}

\section{RÉSUMÉ}

Les résultats d'une recherche doctorale visant à analyser la politique publique en matière d'éducation concernant le travail d'enseignant construit par la dictature civilo-militaire au Chili (1973-1990) sont présentés. Des hypothèses ont été élaborés pour souligné l’importance que le régime a donné aux enseignants par le biais de réglementations juridiques et d'actions 
répressives. Les contributions théoriques de A. Gramsci et d'E. P. Thompson ont facilité le travail d'interrogation de différentes sources, privilégiant parmi elles la législation et les rapports relatifs aux Droits de l'Homme. Les conclusions confirment les hypothèses avancées et ajoutent de nouveaux éléments invitant à revoir les transformations réalisées au cours de cette période sur l'activité des enseignants et le système éducatif chilien en général.

Mots-clés: travail d'enseignant, Dictature Civique-Militaire, Chili, Politiques Publiques Éducatives. 


\section{INTRODUCCIÓN}

Se presentan aquí los resultados finales de una investigación doctoral que tuvo como objeto de estudio la configuración histórica del trabajo docente durante la Dictadura Cívico-Militar en Chile (1973-1990). Dentro de dicho problema y periodo interesó indagar en las diferentes Políticas Públicas que la Dictadura Cívico-Militar construyó sobre el profesorado, tanto desde el punto de vista de la regulación jurídica oficial, como así también, desde las iniciativas realizadas fuera del ámbito de la legalidad a través de la represión política llevada adelante por agentes del Estado. Desde este punto de vista, se trabajó con una comprensión ampliada de la acción del Estado a través de las Políticas Públicas, donde se consideró la acción pública y la acción no pública del mismo sobre el profesorado, posibilitando el desarrollo de una investigación que emprendiera un abordaje entrelazado de estas dos formas de actuar del Estado.

En la bibliografía disponible sobre la educación durante la Dictadura Cívico-Militar en Chile no se ha considerado como un problema de estudio central a la acción del Estado sobre el trabajo docente desde esta perspectiva ampliada, puesto que se ha privilegiado el desarrollo de estudios centrados en los procesos de transformación estructural del sistema educativo (CASTRO, 1977; BERCHENKO, 1983; NÚÑEZ, 1982, 1989, 1990, 2003; PIIE, 1984; COX y GYSLING, 1990; LATORRE, NÚÑEZ, GONZÁLEZ y HEVIA, 1991; ESPINOZA y GONZÁLEZ, 1993; OLIVA, 1998, 2010; COX, 2003; MALDONADO, 2003; RUIZ, 2010; RETAMAL, 2013; BELLEI, 2015) o en aspectos más específicos del trabajo docente como los impactos de la transformación económica neoliberal en la vida laboral (ADLER LOMNITZ y MELNICK, 1998), la violencia política (SÁNCHEZ, 2013) y la organización sindical (REYES, 2005; CANDINA, 2014). Considerando aquello, la investigación que aquí se presenta apuntó a abordar este problema de estudio desde una perspectiva integradora de la acción del Estado. Para lograr aquello, se consideraron las siguientes preguntas orientadoras del trabajo de investigación: ¿Cuáles son las Políticas Públicas Educacionales sobre el trabajo docente en Chile construidas en este periodo? 
¿Qué características tienen estas Políticas Públicas? ¿Qué cambios y continuidades son posibles de identificar en la Política Pública sobre el trabajo docente en este periodo? ¿Qué grupos hicieron estas Políticas Públicas y cuáles eran sus fundamentos políticos, filosóficos e ideológicos?

\section{OPCIONES METODOLÓGICAS Y TEÓRICAS}

El 21 de septiembre de 1973 la Dictadura Cívico-Militar disolvió al Congreso Nacional mediante el Decreto Ley No 27 (CHILE, 1973b). Hacia junio de 1974 la Junta Militar de Gobierno² se arrogó los poderes Constituyente, Legislativo y Ejecutivo a través del Decreto Ley No 527 (CHILE, 1974a), situación que permaneció así hasta el final de la misma. De esta manera, para identificar y analizar las Políticas Públicas Educacionales de la Dictadura CívicoMilitar chilena fue necesario trabajar sobre una tipología de fuentes muy específicas: aquellas producidas en el entorno de producción legislativa del periodo. De esta forma, se analizaron Documentos Estratégicos (JUNTA NACIONAL DE GOBIERNO, 1974; CHILE, 1975; PINOCHET, 1979), Documentos Jurídicos y las Actas de la Junta Militar de Gobierno3, lo que permitió acceder a las ideas que orientaron las líneas generales de la Dictadura Cívico-Militar, a los productos del trabajo legislativo y al contexto de producción de los mismos. Para abordar la violencia política ejercida sobre el profesorado por parte de los agentes de la Dictadura Cívico-Militar se abordaron los informes de violaciones a los derechos humanos elaborados en diferentes momentos del periodo de transición a la democracia (COMISIÓN NACIONAL DE VERDAD Y RECONCILIACIÓN, 1991; COMISIÓN NACIONAL SOBRE LA

\footnotetext{
2 La Junta Militar de Gobierno fue la instancia de Gobierno que se entregó la Dictadura CívicoMilitar después del Golpe de Estado de 1973. Estuvo compuesta por el grupo de líderes del Ejercito, Marina, Fuerza Aérea y Carabineros que se tomaron el poder mediante el Golpe de Estado y centralizó los poderes Ejecutivo, Legislativo y Constituyente.

3 La legislación de la República de Chile y las Actas de la Junta Militar de Gobierno se encuentran disponibles en el Archivo Digital Biblioteca del Congreso Nacional de Chile (https://www.ben.cl/).
} 


\section{PRISIÓN POLÍTICA Y TORTURA, 2005).}

Estas fuentes fueron analizadas tanto desde el punto de vista de su contenido temático propio, como así también, en diálogo con otras Políticas Públicas más amplias emprendidas por la Dictadura Cívico-Militar, puesto que se asumió que las diferentes iniciativas dirigidas hacia el profesorado chileno estaban en diálogo con opciones políticas, económicas e ideológicas de mayor envergadura.

Para desarrollar el proceso de organización y análisis de las diferentes fuentes se tuvo en consideración los aportes teóricos de Edward Palmer Thompson y Antonio Gramsci, fundamentalmente en la línea de contar con una mirada compleja sobre los procesos de disputa del poder en situaciones políticas de alta complejidad. Por lo mismo, durante todo el proceso de desarrollo de esta investigación se tuvo a la vista las conceptualizaciones de Lógica Histórica y Legislación de Edward Palmer Thompson (1981, 1997), como así también las conceptualizaciones de Política y Hegemonía de Antonio Gramsci (1980, 2013). Por Lógica Histórica se ha comprendido a aquella modalidad de investigación histórica que opta por desarrollar una forma de creación de conocimiento desvinculada de preconcepciones teóricas rígidas y que permita llevar adelante un diálogo creativo entre la evidencia histórica y el investigador (Thompson, 1981). Se ha entendido el concepto de Legislación desde la perspectiva de Thompson (1997) como un espacio de disputa de múltiples actores que intentan a través de la misma proyectar sus formas de entender el mundo, evitando adoptar una perspectiva esquemática que identifica a la Ley exclusivamente como una imposición de los más poderosos a los menos poderosos. Si bien Thompson (1997) reconoce que en parte la Ley implica un ejercicio de dominación de clase, a la vez representa un espacio donde la misma dominación debe asumir límites complejos, puesto que de todas maneras debe mostrarse como justa y adecuada. En el caso del concepto de Política en Gramsci (1980, 2013) se ha entendido aquí como el espacio de definición de la distribución de poder entre los diferentes grupos humanos en base a un amplio y complejo 
proceso de enfrentamiento posible de situar en el contexto de las relaciones sociales históricamente determinadas. Con respecto al concepto de Hegemonía en Gramsci $(1980,2013)$ se ha entendido como los esfuerzos desplegados por diferentes grupos organizados tras el objetivo de alcanzar la dirección intelectual y moral de la población mediante el trabajo de diferentes intelectuales. Considerando estas definiciones conceptuales, especialmente las de Lógica Histórica y Legislación, se llevó adelante un proceso amplio de recopilación y análisis de fuentes primarias y secundarias que permitieran dotar a la investigación de un sustento empírico relevante, evitando partir de generalizaciones teóricas predeterminadas y optando por llevar adelante un diálogo creativo en base a diferentes hipótesis. En este ejercicio, teniendo a la mano las conceptualizaciones de Política y Hegemonía, se buscó identificar y analizar en dichas fuentes la diversidad de decisiones tomadas por diferentes actores de la Dictadura Cívico-Militar, poniendo atención especial a los principales lineamientos y conflictos generados en el proceso de producción e implementación de la Política Pública Educacional.

Detrás de estas opciones metodológicas y teóricas se trabajó con un grupo de hipótesis que apuntaban a resaltar que la Dictadura Cívico-Militar le asignó una alta y especial atención al sistema educacional y al trabajo docente a través de la elaboración de regulaciones que modificaron a los mismos; advertir que las regulaciones sobre el trabajo docente fueron diseñadas e implementadas por diferentes actores participes de la Dictadura Cívico-Militar que tenían en común el deseo de transformar el sistema educativo, aunque lo hacían desde diferentes perspectivas teóricas y políticas lo que los hacía enfrentarse y disputar entre sí; indicar que el profesorado fue afectado de manera directa por la violencia política estatal a través de estrategias de vigilancia y persecución especialmente diseñadas por la Dictadura Cívico-Militar para estos fines y proponer que la Política Pública Educacional de la Dictadura Cívico-Militar dirigida al profesorado tendría la capacidad de proyectarse hasta el presente. Estas hipótesis acompañaron y orientaron el proceso de análisis de las fuentes consultadas, permitiendo así desarrollar un diálogo constante con las mismas. 


\section{ENTRE LA RECTIFICACIÓN Y ENALTECIMIENTO AL PROFESORADO (1973-1978) Y LA IMPLEMENTACIÓN DE POLÍTICAS DE MODERNIZACIÓN NEOLIBERAL AUTORITARIAS (1979-1990)}

En el caso de la Política Pública Educacional de la Dictadura CívicoMilitar orientada hacia el profesorado chileno es posible señalar de se identificó dos grandes periodos: uno comprendido entre 1973 y 1978, donde las iniciativas estatales se caracterizaron como poco sistemáticas y contradictorias, puesto que zigzagueaban entre la rectificación y el enaltecimiento hacia el trabajo docente. Por su parte, el segundo periodo comprendido entre 1979 y 1990 se caracterizó por la implementación de políticas de modernización neoliberal autoritarias.

El primer periodo (1973-1978) de la Política Pública Educacional de la Dictadura Cívico-Militar fue liderado por integrantes de la Armada de Chile quienes se ubicaron en diferentes puntos estratégicos de dirección del Ministerio de Educación Pública. Esta presencia de las Fuerzas Armadas fue replicada en los diferentes niveles del sistema educacional a lo largo del país. Uno de los principales lineamientos de la Política Pública Educacional durante este primer periodo fue el desarrollo de diferentes iniciativas de rectificación del profesorado, entendiendo por esto al complejo proceso de intervención de las diferentes instituciones educacionales y la disminución del poder de aquellos segmentos del profesorado identificados como integrantes o simpatizantes del Gobierno de la Unidad Popular liderado por el Presidente Salvador Allende. De esta forma, es posible entender el desarrollo de diferentes iniciativas que buscaron situar en una condición de precariedad a las y los funcionarios públicos mediante el establecimiento de la condición de interinato de su vínculo laboral. El primer movimiento en esta dirección fue la publicación del Decreto Ley $\mathrm{N}^{\circ} 6$ en septiembre de 1973 (CHILE, 1973a) que estableció esta condición y se limitó a indicar la clara posibilidad de cesar de sus puestos de trabajo a diferentes funcionarias y funcionarios públicos mediante reemplazos directos. Los docentes del sistema escolar público gozaban de la condición de empleados 
públicos y vieron como la seguridad de su trabajo se desvanecía a propósito de esta definición de rectificación de carácter político y revanchista. Esta persecución a empleados públicos fue una política recurrente en este periodo y se complementó con diversos instrumentos jurídicos que buscaban entregar más atribuciones al Estado para expurgar a las personas que trabajaban en su interior, tales como: Decreto Ley $\mathrm{N}^{\circ} 22$ de 1973 (CHILE, 1973c); Decreto Ley $\mathrm{N}^{\circ}$ 98 de 1973 (CHILE, 1973d) y el Decreto Ley Nº 2.345 de 1978 (CHILE, 1978b).

Otra iniciativa orientada a la rectificación del profesorado fue el cierre de las Escuelas Normales a través del Decreto Ley $\mathrm{N}^{\circ} 179$ publicado en diciembre de 1973. Las Escuelas Normales eran las instituciones formadoras de docentes de Educación Básica y funcionaban desde mediados del siglo XIX, constituyéndose en una institución con presencia nacional y fundamental en la formación de docentes para el sistema educativo. Desde la mirada de los funcionarios de la Dictadura Cívico-Militar las Escuelas Normales habrían caído en un funcionamiento irregular debido a su baja calidad y complejidad, pero fundamentalmente, debido a que se habrían politizado en demasía, haciéndose parte activa de las disputas político-educacionales del Gobierno de la Unidad Popular. Si bien, al interior de la Junta Militar de Gobierno se habría acordado un cierre y reorganización temporal de las Escuelas Normales4, lo que terminó ocurriendo fue el cierre definitivo de las mismas mediante la publicación del Decreto Ley $\mathrm{N}^{\circ} 179$ en diciembre de 1973. En dicho cuerpo legal se fundamentó la "reorganización de la enseñanza normal" en los siguientes términos:

a) La situación de anarquía en que se desenvuelve la Enseñanza Normal, tanto en sus aspectos técnicos, administrativos y pedagógicos, y que es el propósito de la Junta de Gobierno restablecer los principios de orden, disciplina y moralidad en los establecimientos educacionales, máxime en aquellos que tienen por misión preparar el profesorado de las escuelas de enseñanza básica. (CHILE, 1973f, p. 1).

La tercera iniciativa de carácter rectificador de la Dictadura Cívico-

\footnotetext{
4 Ver Acta de la Sesión Secreta de la Junta Militar de Gobierno del día 31 de Octubre de 1973.
} 
Militar fue el ataque a la principal organización sindical del profesorado, el Sindicato Único de Trabajadores de la Educación (SUTE). En un primer momento la Dictadura Cívico-Militar optó por intervenir al SUTE a través del Decreto Ley No 82 publicado en octubre de 1973. Mediante dicho cuerpo legal se eliminó el financiamiento del SUTE debido a la supresión del descuento mensual por planilla que se realizaba mensualmente a sus afiliados, se congeló el 90\% de sus recursos y se estableció la apertura de procesos de revisión de sus actividades. Estas medidas apuntaban a hacer inviable el funcionamiento del SUTE y evidenciaban una abierta desconfianza hacia su lógica de funcionamiento de parte del régimen. Finalmente, pasados dos años de esta primera medida, a través del Decreto Ley $\mathrm{N}^{0} 1.284$ se materializó la eliminación en el ámbito de la legalidad del SUTE presentándose la siguiente explicación:

$3^{\circ}$.- Que del estudio que se ha hecho de sus actividades se ha podido comprobar que cumplían actividades de carácter gremial y político, que no están permitidos en sus Estatutos, lo que es causal suficiente para proceder a la cancelación de sus personalidades jurídicas. (CHILE, 1975, p. 1).

En este mismo periodo inicial, la Dictadura Cívico-Militar llevó adelante algunas iniciativas que buscaban enaltecer la imagen del profesorado chileno. La primera de estas iniciativas fue la fundación del Colegio de Profesores a través de la publicación del Decreto Ley Nº 678 el 16 de octubre de 1974. Los argumentos utilizados para fundamentar esta nueva institucionalidad fueron presentados en los siguientes considerandos:

Considerando: Que la creación del Colegio de Profesores es una antigua y sentida aspiración del magisterio nacional que ve en ella la forma de conceder a la abnegada labor de profesor la dignificación que el desempeño de su profesión exige. Que cabe destacar que el profesional docente requiere más que ningún otro profesional, de especiales condiciones para el ejercicio de su profesión ya que ésta es una abnegada entrega de lo mejor de sí mismos en bien de los discípulos e implica un constante espíritu de superación para preparar 
a la juventud en los profundos cambios educacionales que vive el país. Que lo anterior ha determinado que el Supremo Gobierno, consciente de la importancia que en el desarrollo social del país tiene el ejercicio de la docencia proceda a crear el Colegio de Profesores como la organización única, profesional y moral que promueva la dignificación del profesor. Que el Colegio de Profesores viene a ser el nuevo cauce a través del cual el profesorado hará valer sus derechos y esta entidad estará llamada a marcar nuevos rumbos en el progreso educacional chileno, y. (CHILE, 1974b, p. 1).

Con la fundación del Colegio de Profesores la Dictadura Cívico-Militar buscaba alejar al profesorado de la lógica de organización de orientación sindicalista que históricamente había construido, puesto que la figura de los Colegios era justamente aquella forma de organización que los grupos profesionales más prestigiosos habían seguido. Por otra parte, con la fundación del Colegio de Profesores la Dictadura Cívico-Militar buscaba controlar la organización del profesorado puesto que puso en su dirección a docentes afines al régimen tales como la profesora Olga Silvia Peña Morales en el periodo 19741979 y el profesor Juan Eduardo Gariazzo Barría en el periodo 1979-1986. Esta situación de falta de autonomía del Colegio de Profesores puso en cuestionamiento su función al interior del profesorado, lo que derivó en una importante falta de apoyo y legitimidad.

La segunda política de enaltecimiento del profesorado fue la Carrera Docente materializada a través del Decreto Ley $\mathrm{N}^{\circ} 2.327$ publicado el 22 de septiembre de 1978. La Carrera Docente vino a atender una demanda importante del profesorado que era contar con una regulación específica para su trabajo, puesto que hasta ese momento el profesorado contaba con una regulación en tanto funcionarios públicos. A través de esta iniciativa se establecieron regulaciones específicas sobre el trabajo del profesorado: condiciones del ejercicio, ingreso a la docencia, nombramientos, destinaciones, ascensos, remuneraciones, horarios, encasillamientos, categorías, entre otras. Así, la Dictadura Cívico-Militar promulgó la Carrera Docente como un instrumento de organización del trabajo de las y los profesores, muy en la línea de las formas tradicionales de regulación propias del Estado Docente, 
entendiendo por esto a un Estado que se preocupa por dotar de caminos y formas de organización de las diferentes instituciones y actores del sistema educacional. No obstante, la Carrera Docente no pudo ser implementada de forma adecuada, tanto por problemas técnicos asociados a las dificultades de traspasar a un grupo laboral numeroso a otra forma de regulación de sus funciones, como así también y de manera fundamental, por dificultades de orientación política, puesto que a contar del año 1979 se comenzó a generar al interior de la Dictadura Cívico-Militar un viraje profundo en sus orientaciones hacia la implementación de políticas marcadas por el pensamiento económico neoliberal, que ameritaban una comprensión de la acción del Estado completamente contraria a las finalidades de la Carrera Docente. De esta forma, de manera paulatina las orientaciones de la Carrera Docente comenzaron a ser abandonadas puesto que dejaron de tener sentido en el contexto mayor de una profunda revolución de corte económico neoliberal autoritario.

El segundo periodo (1979-1990) de la Política Pública Educacional de la Dictadura Cívico-Militar fue liderado por actores civiles de diferente formación y orientación política. Dentro de estos el más significativo fue Gonzalo Vial Correa, historiador y abogado que ocupó el cargo de Ministro de Educación entre el 26 de diciembre de 1978 y el 14 de diciembre de 1979. Se señala que es importante debido a que bajo su mandato se produce un profundo giro en la orientación de la Política Pública Educacional hacia una reestructuración de carácter neoliberal. En marzo de 1979 se hizo público el documento denominado Directiva Presidencial sobre la Educación Nacional (PINOCHET, 1979) donde se señalaron los grandes lineamientos a seguir en la Política Pública Educacional en los siguientes términos: abandono estatal de la responsabilidad de expandir su acción en el sistema educativo abriendo paso a la iniciativa privada; compromiso estatal con el aseguramiento del acceso de la población al nivel de Educación Básica; limitación del acceso a la Educación Media y Educación Superior a un segmento limitado de la población que cumpla con determinada capacidad económica y académica. Estos lineamientos tuvieron repercusión en el espacio destinado a la educación en la Constitución 
Política de la República de Chile creada por la Dictadura Cívico-Militar en Octubre de 1980 (CHILE, 1980c), puesto que allí se reforzó el compromiso del Estado a asegurar exclusivamente el acceso a Educación Básica y a reforzar la noción de Libertad de Enseñanza entendida en términos de la libertad de ofrecer servicios educacionales de diversa naturaleza y el derecho de los padres a escoger la educación de sus hijos.

Considerando lo anterior, se identificó una serie de Políticas Públicas Educativas de orientación neoliberal que afectaron al trabajo docente, tanto en sus espacios de trabajo como en sus espacios de formación. En el caso de los espacios de trabajo del profesorado, la Dictadura Cívico-Militar optó por realizar sendos procesos de descentralización de los Establecimientos Educacionales Fiscales administrados por el Ministerio de Educación Pública. En esta línea se inscribe el proceso de transferencia de Establecimientos de Educación Media Técnico Profesional Públicos a Gremios Empresariales a través del Decreto Ley $\mathrm{N}^{\circ} 3.166$ promulgado en 29 de febrero de 1980 (CHILE, 1980a). Las características de este proceso de transferencia son interesantes de considerar, puesto que se desarrolló mediante la firma de convenios que podían ser prorrogables en el tiempo, no significó gastos importantes para los Gremios Empresariales puesto que el traspaso fue de carácter gratuito y se aseguró un financiamiento público para asegurar el funcionamiento de dichos establecimientos. Para el profesorado que se desempeñaba en estos Establecimientos Educacionales este traspaso significó la pérdida de su condición de empleados públicos puesto que tuvieron que jubilarse o enfrentar el desempleo al perder su vínculo con el Ministerio de Educación Pública. Un segundo proceso de descentralización de los Establecimientos Educacionales Fiscales administrados por el Ministerio de Educación Pública, eso sí de una escala mayor, fue el denominado proceso de Municipalización que implicó el traspaso de los Establecimientos Educacionales Fiscales de Educación Básica y Educación Media a las diferentes Municipalidades del país. De esta forma, a través del Decreto con Fuerza de Ley $\mathrm{N}^{\circ}$ 1-3.063 de junio de 1980 (CHILE, 1980b) se inició el proceso de traspaso de Establecimientos Educacionales 
Fiscales y sus estudiantes a las diferentes Municipalidades de las 336 Comunas existentes en el país en 1980. Solamente en 1980 se contabilizaban 6.370 Establecimientos Educacionales Fiscales de Educación Básica y Educación Media (MALDONADO, 2003) que atendían a cerca de 2.150.338 estudiantes (DÍAZ, LÜDERS y WAGNER, 2016). Ciertamente este proceso de transferencia de Establecimientos Educacionales Fiscales a las Municipalidades tomó un tiempo importante, extendiéndose hasta 1989 , tanto por las complejidades técnicas, políticas y económicas de su materialización. En el caso del profesorado, el proceso de Municipalización provocó efectos muy complejos, en primer lugar, provocó el fracaso en la implementación de la Carrera Docente revisada con anterioridad, puesto que, como segunda consecuencia, el profesorado perdió su condición de empleado público debido a que su actividad comenzó a ser regulada por las mismas condiciones que las y los trabajadores del flexible sistema laboral privado. Vinculado a lo anterior, y a modo de una tercera consecuencia, es posible señalar que el trabajo docente quedó en una situación de abierta exposición al pasar a depender laboralmente de forma directa de las Municipalidades, puesto que las mismas fueron dirigidas durante toda la Dictadura Cívico-Militar por personas de confianza directa del régimen, favoreciéndose así la toma de decisiones en contrataciones y desvinculaciones fundamentadas por cuestiones políticas, como así también, debido al desarrollo de un complejo proceso de desvalorización y diferenciación del salario del profesorado, puesto que éste perdió cualquier regulación sobre su estructuración y límites mínimos, mientras que a la vez, las diferentes Municipalidades remuneraron al profesorado según sus diversas capacidades económicas (ROJAS, 1998), produciéndose así una novedosa y compleja situación de variabilidad y desigualdad salarial poco común en la experiencia del profesorado chileno.

En el caso de las Políticas Públicas Educativas de orientación neoliberal que afectaron los espacios de formación del profesorado destaca la Ley General de Universidades oficializada a través del Decreto con Fuerza de Ley $\mathrm{N}^{\circ} 1$ publicado el o3 de enero de 1981 (CHILE, 1981a). Esta regulación trajo 
transformaciones profundas en el sistema universitario chileno, puesto que propició la incorporación de una nueva comprensión de la perspectiva de Libertad Académica asociada a cuestiones relativas a la propiedad y también incentivó la apertura de nuevas Universidades Privadas lo que vino a transformar el escenario de la Educación Superior chilena al incorporarse nuevos proyectos universitarios vinculados a grupos económicos, religiosos y políticos que anteriormente no tenían dicha representatividad en el espacio universitario. Con respecto al profesorado, la transformación más relevante establecida en el Decreto con Fuerza de Ley $\mathrm{N}^{\circ} 1$ fue el establecimiento de la idea de exclusividad universitaria, la que implicaba que solamente se formaría en las Universidades determinadas profesiones que necesitarían la obtención previa de una Licenciatura Universitaria. Dentro del reducido grupo de 12 Carreras Profesionales 5 no fue incluida la Pedagogía lo que implicaba una evidente pérdida de estatus puesto que no sería desarrollada dentro de la institución educativa de mayor prestigio y desarrollo académico como lo es la Universidad. Esta situación de exclusión de la Pedagogía de la perspectiva de exclusividad universitaria se extendió durante toda la Dictadura Cívico-Militar, tornando profundamente compleja la existencia de la formación docente en el escenario educativo, puesto que como ya fue advertido anteriormente las Escuelas Normales habían dejado de existir. Profesoras y profesores continuaron formándose al interior de diversas Universidades y otro tipo de Instituciones de Educación Superior de menor jerarquía creadas por la misma Dictadura Cívico-Militar sobre la infraestructura física y académica del prestigioso Instituto Pedagógico de la Universidad de Chile en sus Sedes de Santiago y de Valparaíso, ahora separadas de dicha institución ${ }^{6}$. Estas

\footnotetext{
5 Estas Carreras fueron las siguientes: Abogado, Arquitecto, Bioquímico, Cirujano Dentista, Ingeniero Agrónomo, Ingeniero Civil, Ingeniero Comercial, Ingeniero Forestal, Médico Cirujano, Médico Veterinario, Psicólogo y Químico Farmacéutico.

6 Un proceso paralelo de transformación del Sistema de Educación Superior fue la reestructuración de las Universidades Estatales existentes hasta el año 1981: Universidad de Chile y Universidad Técnica del Estado de Chile. A través del Decreto con Fuerza de Ley $\mathrm{N}^{\circ} 2$ (CHILE, 1981b) se estableció que estas Universidades perderían sus sedes regionales, por razones de racionalidad económica y académica, para dar paso a la creación de nuevas instituciones de Educación Superior Estatales, ya sean Universidades, Institutos Profesionales
} 
Instituciones de Educación Superior fueron el Instituto Profesional Academia Superior de Ciencias Pedagógicas de Santiago y el Instituto Profesional Academia Superior de Ciencias Pedagógicas de Valparaíso respectivamente que mantendrían ese nombre entre 1981 y 1985 cuando pasaron a denominarse Universidad Metropolitana de Ciencias de la Educación y Universidad de Playa Ancha de Ciencias de la Educación respectivamente. Estos cambios institucionales se explican por diversas pugnas y presiones en torno al problema de pérdida de prestigio de la formación docente, incluso dentro de la misma Junta Militar de Gobierno no había consenso sobre la exclusión de la Pedagogía de la noción de exclusividad universitaria y sobre el hecho de que la Pedagogía fuese desarrollada en Instituciones de Educación Superior de menor calidad como las Academias Superiores de Ciencias Pedagógicas. En esta línea, la creación de las Universidades Pedagógicas fue un problema ampliamente abordado en las Sesiones Secretas de la Junta Militar de Gobierno y fue a partir de la propuesta de José Toribio Medina, Comandante en Jefe de la Armada de Chile, que se nombrarían como Universidad Metropolitana de Ciencias de la Educación mediante la Ley $\mathrm{N}^{\mathrm{o}} 18.433$ (CHILE, 1985a) y Universidad de Playa Ancha de Ciencias de la Educación mediante la Ley No 18.434 (CHILE, 1985b), ambas en septiembre de 1985 .

Ciertamente que el escenario de Políticas Públicas Educacionales descrito hasta aquí tiene un abierto cariz regresivo, con consecuencias complejas para el trabajo docente, puesto que provocaron una desestructuración profunda de la lógica de relación laboral del profesorado, en un contexto de prohibición de la actividad sindical. En otras palabras, el profesorado vivenció la destrucción de las estructuras de regulación y protección de su actividad laboral sin tener instancias institucionales para intentar poner freno a las mismas. De esta forma, las diferentes problemáticas laborales sufridas por el profesorado fueron ganando una abierta notoriedad, lo

o Centros de Formación Técnica. En este movimiento, la Universidad de Chile perdió al Instituto Pedagógico de Santiago y de Valparaíso, donde se formaban Profesoras y Profesores de Educación Media. 
que fue obligando a la Dictadura Cívico-Militar a proponer alguna respuesta a la situación. Lo interesante de esto es que este problema dio paso a una discusión entre diferentes grupos integrantes de la Junta Militar de Gobierno, puesto que para un sector liderado por las Comisiones Legislativas de la Armada de Chile y de Carabineros de Chile era importante reestablecer algunas regulaciones básicas en torno a la organización del trabajo docente, tales como remuneración mínima para el profesorado que trabajase en Establecimientos Educacionales que recibían financiamiento estatal; disposiciones sobre el ingreso, mantención y salida del ejercicio docente; reducción de las horas de trabajo; entre otras. Mientras que para otro sector, liderado por Augusto Pinochet, el Ministerio de Educación Pública, por las Comisiones Legislativas de la Fuerza Aérea de Chile y del Ejército de Chile, era necesario establecer algunas regulaciones muy básicas que en lo fundamental mantuviesen al trabajo docente tal como se encontraba, o sea, que el profesorado continuara siendo un grupo de trabajadores particulares y que sus remuneraciones se establecieran a través de la lógica de la oferta y la demanda. Finalmente se impuso la segunda perspectiva y en marzo de 1987 se publicó la Ley $\mathrm{N}^{\circ} 18.602$ (CHILE, 1987) que estableció algunas regulaciones básicas que no afectaban la condición de trabajadores privados del profesorado en pleno proceso de implementación del proceso de Municipalización, donde el Ministerio de Educación pudo poner en funcionamiento lógicas de reubicación y desvinculación de docentes a lo largo del país contando con las herramientas flexibles y de bajo costo establecidas en la regulación laboral privada. De esta forma, es posible sostener que esta iniciativa, que vivió un momento inicial de enfrentamiento de posiciones al interior de la Junta Militar de Gobierno, se fue orientando cada vez más a situarse como una herramienta eficaz para legitimar el proceso de reorganización y racionalización de las plantas docentes de los Establecimientos Educacionales Municipalizados y no necesariamente como una herramienta para regular y facilitar el desarrollo del trabajo docente.

Finalmente, en términos de la estructuración más amplia del nuevo sistema educacional construido por la Dictadura Cívico Militar, se publicó en 
marzo de 1990, un día antes de la asunción del primer Gobierno de Transición7, la Ley No 18.962 Orgánica Constitucional de Enseñanza (CHILE, 1990) que cumplió el rol de establecer jurídicamente en un nivel de carácter constitucional las lógicas de funcionamiento neoliberal de la educación: rol del Estado Subsidiario, financiamiento a la demanda vía vouchers, competencia de Establecimientos Educacionales por estudiantes y recursos, entre otras.

\section{LA REPRESIÓN Y VIGILANCIA HACIA EL PROFESORADO (1973-1990)}

La Dictadura Cívico-Militar chilena se caracterizó por llevar adelante un profundo proceso de persecución y exterminio de potenciales opositores políticos. Para lograr esto ocupó una vía jurídica destinada a instaurar toda una legislación que facilitara el control del territorio y su población, como así también, asegurar la hegemonía del uso de la fuerza. No obstante lo anterior, la Dictadura Cívico-Militar también utilizó una vía distinta a la jurídica que tenía que ver con la implementación de acciones posibles de conceptualizar mediante la noción del Terrorismo de Estado, a saber: asesinatos; desapariciones forzadas; torturas; violencia sexual; detenciones injustificadas; vigilancia y amedrentamiento; prisión política; secuestros; confinamientos; allanamientos; exilio; entre otras, cometidas por agentes del Estado y por civiles vinculados al régimen.

Tal como otros grupos laborales, el profesorado fue duramente castigado mediante diferentes modalidades represivas. En el Informe de la Comisión Nacional de Verdad y Reconciliación publicado el o8 de febrero de 1991 (COMISIÓN NACIONAL DE VERDAD Y RECONCILIACIÓN, 1991) que tenía como finalidad indagar y sistematizar las más graves violaciones a los Derechos Humanos, Pamela Sánchez (2013) logró identificar a 64 Profesoras y

\footnotetext{
7 El 11 de marzo de 1990 asumió la Presidencia de la República de Chile el abogado vinculado al Partido Demócrata Cristiano Patricio Aylwin Azócar, siendo el primer Presidente electo democráticamente en el periodo conocido como Transición a la Democracia.
} 
Profesores Detenidos Desaparecidos y 70 Detenidos Ejecutados. Con el objetivo de señalar un perfil del profesorado víctima del Terrorismo de Estado se pueden identificar como las más representativas las siguientes características: ser mayoritariamente hombres jóvenes y de mediana edad; sostener una militancia política abiertamente reconocida; ser en su mayoría de nacionalidad chilena; ser desaparecidos y ejecutados de forma mayoritaria en los primeros momentos de existencia de la Dictadura Cívico-Militar (1973-1974) y de manera más recurrente en la ciudad de Santiago. Este perfil es coherente con la imagen completa del Terrorismo de Estado diseñado e implementado por la Dictadura Cívico-Militar, puesto que la violencia estatal con apoyo civil fue dirigida fundamentalmente hacia personas identificadas con proyectos de transformación social afines a los Partidos Políticos de izquierda vinculados al Gobierno de la Unidad Popular. En esa perspectiva, las y los profesores que fueron participes entusiastas de dicho proyecto de transformación social y política, desde el mundo educacional, fueron identificados como opositores desde la perspectiva de las nuevas autoridades, lo que justificaba su persecución, vigilancia y potencial eliminación.

Las técnicas represivas utilizadas contra el profesorado chileno incluyeron, además de la ejecución y desaparecimiento, otras vías de acción. Dentro de estas resalta la vigilancia como una opción privilegiada. La vigilancia constante se constituyó en una herramienta de uso cotidiano para conocer y administrar políticamente al profesorado. El Ministro de Educación Pública, Contraalmirante Hugo Castro Jiménez, según registros del Acta $\mathrm{N}^{\circ} 160$ A de la Sesión Secreta de la Junta Militar de Gobierno, del día o8 de octubre de 1974, asume abiertamente el activo papel que cumplía el trabajo de Inteligencia como uno más de los diferentes actores que influían en el sistema educacional chileno:

También tiene incidencia la parte seguridad del país. La Inteligencia está permanentemente funcionando, dando datos, indicando que hay que sacar a determinado profesor, que hay que poner a otro, etc. El Ejército coopera mucho; Carabineros en menor escala; la Armada y la FACH también tienen delegados y en algunas provincias tienen más 
cantidad de gente que el Ejército; pero el Ejército a través de los Intendentes influye bastante. (JUNTA DE GOBIERNO, 1974, p. 2).

De esta forma, la vigilancia se constituyó en una tarea de amplia importancia, puesto que servía como vía para identificar, controlar y castigar a docentes opositores al régimen. De esta forma, en la revisión de las Actas de la Junta Militar de Gobierno aparecen instituciones y actores educativos como objetos constantes de vigilancia y persecución, tanto de forma directa, como así también a través de la implementación de otras Políticas Públicas Educacionales como las ya señaladas Carrera Docente y Municipalización, mediante las cuales docentes identificados como opositores eran marginados o desvinculados de sus puestos de trabajo. Un ejemplo de esto se encuentra en la discusión de la implementación de la Carrera Docente (1978-1981) donde el Ministro de Educación Pública, Alfredo Prieto Bafalluy, entregó argumentos al General Augusto Pinochet Ugarte, en la Sesión Secreta de la Junta Militar de Gobierno del día 24 de abril de 1980, defendiendo la necesidad de que sean aprobadas algunas modificaciones a la Carrera Docente con miras a facilitar el despido de determinado perfil de profesoras y profesores:

EL GENERAL PINOCHET, PRESIDENTE DE LA REPUBLICA. (No se entiende el comienzo de la frase)... usted se está preparado para el futuro. EL señor PRIETO, MINISTRO DE EDUCACION,Exactamente. Si me permite, señor Presidente, quiero señalar que la facultad de desburocratización, que la estamos usando en el Ministerio para algunos casos, puede ser puntual, porque yo no puedo sacar a 1,000 profesores con esta facultad, pero sí puedo utilizar esta herramienta para la reducción de jornada; tomar a aquellos cuya labor, hoy día, es nada más que hacer activismo en contra del Gobierno por nuestro sistema educacional. Es una facultad que debemos usar, porque estamos cometiendo un haraquiri con nuestro campo de la educación. Quiero señalar, a título de ejemplo, que tengo aquí en mi poder, determinados en lista, hechos de trabajo detectados con el servicio de inteligencia de toda institución, fundamentalmente, el C.N.I. y comprobados, respecto solamente a profesores que realizan actividades, excluyo a todo aquel profesor que no sea marxista, sino solamente clasificados entre comunistas, socialistas, miristas y marxistas; he excluido a todos los que son demócratas y que están en 
contra del Gobierno, y dentro de esos, sólo aquellos que están realizando activismo en contra del Gobierno en la sala. Es una lista por región y por escuela que sobrepasa los 3 mil a 4 mil profesores. Esa gente no la podemos tener dentro del sistema y para mí, el no tener esta facultad del titulado, me amarra las manos para poder, de alguna manera, tomar medidas con ellos. Esos son datos que tiene el C.N.I., que tienen los dirigentes... (JUNTA DE GOBIERNO, 1980, p. 52-53).

A partir de la presentación del Ministro de Educación Pública, queda clara la utilización política de la Carrera Docente como una vía posible para vigilar y perseguir a docentes identificados como opositores a la Dictadura Cívico-Militar. Para realizar esto ciertamente era preciso contar con procedimientos y herramientas de categorización del profesorado según orientación y acción política a nivel nacional, tarea solo posible de ser realizada por los organismos represivos ${ }^{8}$ que contaban con la capacidad técnica y logística para aquello. En las palabras de Prieto Bafalluy se habla de cerca de cuatro mil docentes izquierdistas identificados a nivel nacional, pudiendo afirmar así que el ojo y oído vigilante de la Dictadura Cívico-Militar tenían la capacidad de extenderse a lo largo y ancho de Escuelas y Liceos a nivel nacional.

Un elemento central del funcionamiento de los ejercicios de vigilancia sobre el profesorado era la delación. Esta fue incentivaba por el régimen mediante diversos Bandos Militares ${ }^{9}$ que terminaron incluyendo la opción de entregar incentivos económicos a las personas delatoras:

12.- Se acuerda reactualizar el Bando que ofrece recompensas a quien entregue antecedentes sobre activistas buscados. Se instruye al señor Ministro del Interior al respecto. (Junta de Gobierno, 1973, p. 2).

\footnotetext{
8 La Dictadura Cívico-Militar contó con dos organismos represivos integrados por miembros de las diferentes Fuerzas Armadas y de Orden, como así también por civiles, encargados de llevar a cabo las operaciones de inteligencia y represión a personas identificadas como opositoras al régimen. Estos organismos represivos fueron: a) la Dirección de Inteligencia Nacional (DINA) que estuvo operativa entre 1973 y 1977; b) la Central Nacional de Informaciones (CNI) que estuvo operativa entre 1977 y 1990.

9 Los Bandos Militares fueron mandatos construidos por la Dictadura Cívico-Militares a partir del Golpe de Estado del 11 de septiembre de 1973 dirigidos hacia la población con la finalidad de informar diferentes medidas y exigir la realización de determinadas acciones. Los Bandos Militares eran comunicados a través de radios y periódicos.
} 
Con miras a organizar y sistematizar la recepción de informaciones asociadas al sistema educacional, funcionó en el Ministerio de Educación Pública una Oficina de Seguridad que tuvo como una de sus funciones principales gestionar diferentes situaciones vinculadas a las tareas de vigilancia y persecución política dirigidas a personas ubicadas en los Establecimientos Educacionales de Educación Básica, Media y Superior, como así también, a personas que trabajaban en la administración del sistema educacional. Lo que muestran las fuentes consultadas es la existencia de una amplia red de comunicación entre el aparato de represión y diversas autoridades de la administración pública, lo que permitía la realización de diversas acciones de consulta y registro de antecedentes de docentes, como así también, en los casos que fuese necesario, realizar acciones de recopilación de más antecedentes o expulsión de docentes de sus puestos de trabajo por cuestiones políticas. En la revisión de las fuentes es interesante notar una activa participación de padres, apoderados, estudiantes, docentes y otros actores en acciones de delación y acusación de participación en actividades de orientación política de profesoras y profesores. De esta forma, se destaca aquí que el funcionamiento de toda la maquinaria represiva en el sistema educacional contó con apoyo de diversos actores civiles simpatizantes de la Dictadura Cívico-Militar, incluidos dentro de estos también a sectores del profesorado.

\section{CONCLUSIONES}

Las conclusiones son organizadas aquí en diálogo con las cuatro hipótesis que guiaron esta investigación.

La primera hipótesis apuntó a destacar que la Dictadura Cívico-Militar prestó una especial atención al sistema educacional y al trabajo docente mediante la elaboración de regulaciones que modificaron a los mismos. Esto fue ampliamente corroborado, puesto que el régimen presentó una porfiada y reiterativa inclinación por crear y dirigir nuevas regulaciones sobre el 
profesorado. Además, la Dictadura Cívico-Militar fue exitosa en su objetivo de transformar el trabajo docente, tanto en su naturaleza jurídico/laboral; su organización gremial; su sistema de formación inicial docente; sus remuneraciones; sus espacios de desempeño profesional; entre otros. Esto produjo un complejo proceso de destrucción de derechos y protecciones alcanzadas por el profesorado en décadas de lucha y organización, tales como la pérdida del valor de su trabajo; la exposición al Mercado; el deterioro de su actuación político/cultural en la sociedad chilena. Desde este punto de vista, las Políticas Públicas Educacionales de la Dictadura Cívico-Militar orientadas hacia el trabajo docente se constituyeron en una herramienta política eficaz en el establecimiento de una nueva relación de poder entre el Estado y el grupo laboral del profesorado, donde el primero consiguió establecer un nivel de alta influencia y acción sobre el segundo, eliminando contrapesos mínimos que posibilitaran tener una relación equilibrada. Ciertamente, que en una dimensión más amplia esto está vinculado a la transformación del Estado chileno, que transitó de la imagen del Estado Docente al Estado Subsidiario.

La segunda hipótesis apuntaba a destacar que las Políticas Públicas Educacionales fueron elaboradas por diferentes actores de la Dictadura CívicoMilitar, que tenían disímiles perspectivas teórico/políticas y compartían el objetivo de transformar lo existente. En la investigación se constató la existencia de variados actores que actuaron en la creación e implementación de las nuevas regulaciones dirigidas hacia el trabajo docente, a saber: militares, abogados, economistas, funcionarios públicos, docentes, entre otros, que movilizaron perspectivas diferenciadas desde el punto de vista teórico, político y valórico, posibles de ser caracterizadas de anti-marxistas, nacionalistas, corporativistas, neoliberales, católicas, de seguridad nacional, entre otras. Sobre esto es posible agregar que efectivamente el trabajo docente fue exitosamente transformado mediante la acción de estos diferentes actores. Sin embargo, si bien es innegable el éxito en la tarea de transformar el trabajo docente conocido hasta 1973, como así también el sistema educacional en su conjunto, también es innegable que no necesariamente los diferentes actores con sus referentes teóricos, políticos y 
valóricos diversos trabajaron en un contexto de total armonía y coherencia de perspectivas, especialmente en el periodo comprendido entre 1973-1978 donde las orientaciones de carácter neoliberal tuvieron poca presencia en las Políticas Públicas Educacionales, como así también, eran resistidas e intensamente criticadas por diversos actores al interior de los espacios de decisión de la Junta Militar de Gobierno. Desde este punto de vista, es posible señalar que la Dictadura Cívico-Militar tuvo serias dificultades para articular un lineamiento hegemónico en la organización y dirección de las diferentes tendencias que la componían. Esto queda claro al identificar Políticas Públicas Educacionales contradictorias entre sí o desprovistas de amplios consensos, especialmente en los primeros años de existencia de la Dictadura Cívico-Militar. Si bien, desde 1979 en adelante las propuestas neoliberales consiguen establecerse como la orientación fundamental de la Política Pública Educacional, llama la atención que solamente en los últimos días de su existencia la Dictadura Cívico-Militar haya conseguido aunar un consenso interno para sancionar la Ley Orgánica Constitucional de Enseñanza $\mathrm{N}^{\circ} 18.962$ (CHILE, 1990) que finalmente instauró de forma amplia y estable esta perspectiva de pensamiento económico como estructura de organización del sistema educativo chileno.

La tercera hipótesis indicaba que el trabajo docente fue afectado por la violencia política a través de estrategias de vigilancia y persecución especialmente diseñadas por la Dictadura Cívico-Militar para el profesorado. En el desarrollo de la pesquisa esto fue corroborado, puesto que dicha violencia política existió con dichas características, como así también se sustentó en el poderío y capacidad técnica de complejas organizaciones de inteligencia y represión política, que contaban con amplias capacidades materiales y humanas para emprender sus actividades. Se sostiene además que esta violencia política se orientó con mayor intensidad hacia un perfil de docente específico: mayoritariamente hombres jóvenes y de mediana edad; que sostenían una militancia política abiertamente reconocida; ser en su mayoría de nacionalidad chilena; ser desaparecidos y ejecutados de forma mayoritaria en los primeros momentos de la Dictadura Cívico-Militar (1973-1974) y de manera más 
recurrente en la ciudad de Santiago. Además de esto, fue posible reconstruir diferentes informaciones que permiten sostener que efectivamente el trabajo docente y sus especificidades, obligaron a la Dictadura Cívico-Militar a desplegar todo un aparataje para hacer viables sus tareas de represión y vigilancia hacia el profesorado. En esta línea, fue posible identificar la poderosa capacidad de influencia en el sistema educacional del aparato represivo del régimen, tanto a nivel de los amplios canales de comunicación y coordinación existentes entre importantes funcionarios públicos del Ministerio de Educación Pública y los líderes de los organismos de represión; como así también, a través de la existencia de complejos procesos de entrecruzamiento de usos y objetivos en las Políticas Públicas Educacionales dirigidas hacia el trabajo docente. Desde un punto de vista político estos hallazgos son interesantes, puesto que indican que pese a todos los esfuerzos desplegados por la Dictadura Cívico-Militar para atraer al profesorado hacia su proyecto de país y de educación no habría alcanzado resultados positivos, puesto que optó con mayor decisión por el uso de la fuerza abierta y velada para controlar al profesorado. De esta forma, habría primado en la acción de la Dictadura Cívico-Militar hacia el profesorado la coacción por sobre el convencimiento. Este hallazgo debe ser complementado con futuras investigaciones que indaguen de forma específica en el apoyo civil que recibió la Dictadura Cívico-Militar en Chile, especialmente el apoyo de sectores del profesorado que en esta investigación fue posible identificar de manera acotada y puntual.

Finalmente, la cuarta hipótesis de esta investigación indicaba que la Política Pública Educacional de la Dictadura Cívico-Militar relacionada al trabajo docente se proyecta con fuerza y claridad hacia el presente. Esta hipótesis se sostiene debido a que la Dictadura Cívico-Militar alcanzó una posición hegemónica en torno al establecimiento del Modelo Neoliberal como ideología fundamental de organización del sistema educacional, que ha tenido en los años posteriores la capacidad de imponer una sobrevalorización del mercado y la negación de la política en el funcionamiento del mismo. Esto se manifiesta de manera clara en diferentes fenómenos contemporáneos: primero: 
la capacidad que el Modelo Neoliberal ha demostrado para irradiar su influencia a lo largo y ancho de la sociedad chilena, incluyendo por cierto a parte importante de la Clase Política Profesional que se articuló en los difíciles días de oposición a la Dictadura Militar. Segundo: articulado con lo anterior, las Políticas Públicas Educacionales creadas durante la Dictadura Cívico-Militar continúan gozando de muy buena salud hasta el presente, en tanto aquellas regulaciones más significativas se constituyen aún en la viga maestra de todo el sistema educacional más allá de algunas transformaciones menores. Tercero: el profesorado experimenta un proceso diferenciado de luchas, marcadas por la herencia de la Dictadura Cívico-Militar y la emergencia de un constante asedio de nuevas regulaciones y exigencias, especialmente de parte de organismos internacionales y de parte de cierta intelectualidad educacional profundamente influida por viejas y nuevas elaboraciones del Modelo Neoliberal. Cuarto: la presencia de las Políticas Públicas Educacionales de la Dictadura Cívico-Militar hacia el trabajo docente, hacia el sistema educacional en su conjunto, como así también, las diferentes Políticas Públicas implementadas en el contexto de la instauración del Modelo Neoliberal en Chile, se muestran profundamente difíciles de transformar en el presente.

\section{REFERENCIAS}

ADLER LOMNITZ, L.; MELNICK, A. Neoliberalismo y Clase Media: el caso de los profesores de Chile. Santiago: DIBAM-Centro de Investigaciones Diego Barros Arana, 1998. 163 p.

BELLEI, C. El gran experimento. Mercado y privatización de la educación chilena. Santiago: LOM, 2015. 254 p.

BERCHENKO, P. Populismo y autoritarismo: alternativas escolares en Chile, 1964-1977. Perpiñán: Universidad de Perpiñán, 1983. 278 p.

CANDINA, A. Vivir una dictadura: historia y memoria de los profesores en Chile (1973-1990). Historia 396, Valparaíso, n. 2, p. 187-216, 2014. 
CASTRO, P. La Educación en Chile de Frei a Pinochet. Salamanca: Ediciones Sígueme, 1977. 237p.

CHILE. Decreto Ley $\mathrm{N}^{\circ} 6$ Declara en calidad de interino los personales que indica. Ministerio del Interior y Subsecretaría del Interior, Santiago, 19 Septiembre 1973a. Disponible en: http://bcn.cl/1jrud Consultado en: 14 Julio 2015 .

CHILE. Decreto Ley $\mathrm{N}^{\circ} 27$ Disuelve el Congreso Nacional. Ministerio de Justicia, Santiago, 24 Septiembre 1973b. Disponible en: http://bcn.cl/1mdox Consultado en: 14 Julio 2015.

CHILE. Decreto Ley $\mathrm{N}^{\circ} 22$ Complementa y aclara el Decreto Ley $\mathrm{N}^{\circ}$ 6, de 12 de Septiembre de 1973. Ministerio del Interior y Subsecretaría del Interior, Santiago, 02 Octubre 1973c. Disponible en: http://bcn.cl/1xirp Consultado en: 14 Julio 2015.

CHILE. Decreto Ley $\mathrm{N}^{\circ} 98$ Declara en reorganización todos los servicios de la administración pública, organismos e instituciones que indica. Ministerio de Justicia, Santiago, 26 Octubre 1973d. Disponible en: http://bcn.cl/1rnfw Consultado en: 14 Julio 2015.

CHILE. Decreto Ley N ${ }^{\circ} 82$ Suspende Aplicación del Artículo $3^{\circ}$ de la Ley 17.615, de 1972. Ministerio de Educación Pública, Santiago, 31 Octubre 1973e. Disponible en: http://bcn.cl/1jqwb Consultado en: 14 Julio 2015.

CHILE. Decreto Ley N ${ }^{\circ} 179$ Declara en reorganización la Enseñanza Normal chilena. Ministerio de Educación Pública, Santiago, 13 Diciembre 1973f. Disponible en: http://bcn.cl/1jqxb Consultado en: 14 Julio 2015.

CHILE. Decreto Ley No 527 Aprueba Estatuto de la Junta de Gobierno. Ministerio del Interior, Santiago, 26 Junio 1974a. Disponible en: http://bcn.cl/1mhkj Consultado en: 14 Julio 2015.

CHILE. Decreto Ley $N^{\circ} 678$ Crea el Colegio de Profesores de Chile. Ministerio de Educación Pública, Santiago, 16 Octubre 1974b. Disponible en: http://bcn.cl/p6nu Consultado en: 14 Julio 2015.

CHILE. Objetivo Nacional del Gobierno de Chile. Santiago: s/i, 1975. 47 p.

CHILE. Decreto Ley $\mathrm{N}^{\circ}$ 1.284 Cancela Personalidad Jurídica a los Organismos que indica y crea Comisión Administradora de sus Bienes. Ministerio de Educación Pública, Santiago, 19 Diciembre 1975. Disponible en: http://bcn.cl/1h2xs Consultado en: 14 Julio 2015. 
CHILE. Decreto Ley N ${ }^{\circ}$ 2.327 Crea la Carrera Docente y regula su ejercicio. Ministerio de Educación Pública, Santiago, 22 Septiembre 1978a. Disponible en: http://bcn.cl/3i2a Consultado en: 14 Julio 2015.

CHILE. Decreto Ley $\mathrm{N}^{\circ}$ 2.345 Otorga facultades al Ministro del Interior respecto de materias que indica. Ministerio del Interior, Santiago, 20 Octubre 1978b. Disponible en: http://bcn.cl/1xk9v Consultado en: 14 Julio 2015.

CHILE. Decreto Ley $\mathrm{N}^{\circ}$ 3.166 Autoriza entrega de la administración de determinados Establecimientos de Educación Técnico Profesional a las instituciones o a las personas jurídicas que indica. Ministerio de Educación Pública, Santiago, o6 Febrero 1980a. Disponible en: http://bcn.cl/12sh Consultado en: 14 Julio 2015.

CHILE. Decreto con Fuerza de Ley $\mathrm{N}^{\circ}$ 1-3063 reglamenta aplicación inciso segundo del artículo $38^{\circ}$ del DL N ${ }^{\circ} 3.063$, de 1979. Ministerio del Interior, Santiago, 13 Junio 1980b. Disponible en: http://bcn.cl/pmd Consultado en: 14 Julio 2015 .

CHILE. Decreto Supremo No 1.150 Constitución Política de la República de Chile. Ministerio del Interior, Santiago, 24 Octubre 1980c. Disponible en: http://bcn.cl/1wb8d Consultado en: 14 Julio 2015.

CHILE. Decreto con Fuerza de Ley $\mathrm{N}^{\circ} 1$ Fija Normas sobre Universidades. Ministerio de Educación Pública, Santiago, o3 Enero 1981a. Disponible en: http://bcn.cl/12rb Consultado en: 14 Julio 2015.

CHILE. Decreto con Fuerza de Ley $\mathrm{N}^{\circ} 2$ Fija Normas sobre Universidades. Ministerio de Educación Pública, Santiago, o7 Enero 1981b. Disponible en: http://bcn.cl/1lts Consultado en: 14 Julio 2015.

CHILE. Ley $\mathrm{N}^{\circ}$ 18.433 Crea la Universidad Metropolitana de Ciencias de la Educación. Ministerio de Educación Pública, Santiago, o4 Septiembre 1985a. Disponible en: http://bcn.cl/27wg Consultado en: 14 Julio 2015.

CHILE. Ley $\mathrm{N}^{\circ}$ 18.434 Crea la Universidad de Playa Ancha de Ciencias de la Educación. Ministerio de Educación Pública, Santiago, 04 Septiembre 1985b. Disponible en: http://bcn.cl/27wk Consultado en: 14 Julio 2015.

CHILE. Ley $\mathrm{N}^{\circ} 18.602$ Normas especiales para personal docente que indica. Ministerio de Educación Pública, Santiago, 23 Febrero 1987. Disponible en: http://bcn.cl/1v4dc Consultado en: 14 Julio 2015.

CHILE. Ley Orgánica Constitucional de Enseñanza Nº 18.962. Ministerio de Educación Pública, Santiago, 10 Marzo 1990. Disponible en: http://bcn.cl/1p80 
Consultado en: 14 Julio 2015.

COMISIÓN NACIONAL DE VERDAD Y RECONCILIACIÓN. Informe de la Comisión Nacional de Verdad y Reconciliación. Santiago: Secretaria General de Gobierno - Secretaría de Comunicación y Cultura, 1991, 2 Tomos 3 Volúmenes.

COMISIÓN NACIONAL SOBRE LA PRISIÓN POLÍTICA Y TORTURA. Informe de la Comisión Nacional Sobre la Prisión Política y Tortura. Santiago: La Comisión, 2005, 777 p.

COX, C.; GYSLING, J. La formación del profesorado en Chile 18421987. Santiago: CIDE, 1990. 321 p.

COX, C. Políticas Educacionales en el Cambio de Siglo. La reforma del sistema escolar en Chile. Santiago: Editorial Universitaria, 2003. 705 p.

DÍAZ, J.; LÜDERS. R.; WAGNER, G. Chile 1810 - 2010. La República en cifras. Historical statistics. Santiago: Ediciones Universidad Católica de Chile, 2016, $780 \mathrm{p}$.

ESPINOZA, O; GONZÁLEZ, L. La experiencia del proceso de desconcentración y descentralización educacional en Chile 19741989. Santiago: PIIE, 1993. $267 \mathrm{p}$.

FAUNES, M. Aulas que permanecerán vacías. Santiago: Cuarto Propio, 2008. $310 \mathrm{p}$.

GRAMSCI, A. Notas sobre Maquiavelo, sobre la política y sobre el Estado moderno. Madrid: Ediciones Nueva Visión, 1980. 317 p.

GRAMSCI, A. Antología. Madrid: Ediciones Akal, 2013. 471 p.

JUNTA DE GOBIERNO. Actas Junta de Gobierno. Santiago, Acta $\mathrm{N}^{\circ}$ 24, Sesión Secreta 23 Octubre 1973. Página 2.

JUNTA DE GOBIERNO. Actas Junta de Gobierno. Santiago, Acta $\mathrm{N}^{\circ} 160 \mathrm{~A}$, Sesión Secreta o8 Octubre 1974. Página 2.

JUNTA DE GOBIERNO. Actas Junta de Gobierno. Santiago, Acta Nº 392 A, Sesión Secreta 24 Abril 1980. Página 52-53.

JUNTA NACIONAL DE GOBIERNO. Declaración de Principios del Gobierno de Chile. Santiago: Junta Nacional de Gobierno, 1974. 38 p. LATORRE, C.; NÚÑEZ, I.; GONZÁLEZ, L.; HEVIA, R. La Municipalización 
de la Educación: una mirada desde los administradores del sistema. Un estudio a nivel comunal. Santiago: PIIE, 1991. $172 \mathrm{p}$.

MALDONADO, M. La privatización de la educación en Chile. San José: Internacional de la Educación, 2003, 66 p.

NÚÑEZ, I. Cambios en la situación del magisterio. Santiago: PIIE, 1982. $81 \mathrm{p}$.

NÚÑEZ, I. Historia del trabajo docente y formación de profesores en Chile. Santiago: PIIE, 1989. 34 p.

NÚÑEZ, I. Reformas educacionales e identidad de los docentes. Chile, 1960-1973. Santiago: PIIE, 1990. 251 p.

NÚÑEZ, I. La ENU entre dos siglos. Ensayo histórico sobre la Escuela Nacional Unificada. Santiago: LOM, 2003. 144 p.

OLIVA, M. Política educativa y profundización de la desigualdad en Chile. Estudios Pedagógicos, v. 34, n.2, p. 207-226, 1998.

OLIVA, M. Política educativa chilena 1965-2009. ¿Qué oculta esa trama? Revista Brasileira de Educação, v. 15, n. 44, p. 311-328, 2010.

PIIE. Las Transformaciones Educacionales bajo el Régimen Militar. (2 volúmenes). Santiago, Chile: Programa Interdisciplinario de Investigación en Educación, 1984. 652 p.

PINOCHET, A. Directiva presidencial sobre la educación nacional. Separata publicada en Cuaderno del Profesor Rural, Santiago, n. 17, p. IVIII, mayo-junio 1979.

RETAMAL, J. Nos siguen pegando abajo. Jaime Guzmán, dictadura, concertación y alianza: 40 años de educación de mercado. Santiago: Ceibo Ediciones, 2013. $87 \mathrm{p}$.

REYES, Leonora. Movimientos de educadores y construcción de política educacional en Chile (1921-1932 y 1977-1994). 2005. 300 p. Tesis (Doctorado en Historia) - Facultad de Filosofía y Humanidades, Universidad de Chile, Santiago, 2005.

ROJAS, P. Remuneraciones de los profesores en Chile. Estudio Públicos, Santiago, n. 71, p. 121-175, 1998.

RUIZ, C. De la República al mercado. Ideas educacionales y política en Chile. Santiago: LOM, 2010. 174 p. 


\section{SÁNCHEZ, P. Una asignatura pendiente: profesores y profesoras} detenidos/as desaparecidos/as y ejecutados/as en Chile de 1973 a 1990. Santiago: Editorial ARCIS, 2013. 423 p.

THOMPSON, E. P. A Miséria da teoria ou um planetário de erros. Uma crítica ao pensamento de Althusser. Rio de Janeiro: Zahar Editores, 1981. $230 \mathrm{p}$.

THOMPSON, E. P. Senhores e Caçadores: a Origem da Lei Negra. Rio de Janeiro: Paz e Terra, 1997. 432 p.

FELIPE ANDRES ZURITA GARRIDO é Doutor em Educação (Universidade Federal de Minas Gerais, 2017). Magister em Educação (Universidade Academia de Humanismo Cristiano, 2012). Professor de Historia e Ciências Sociais (Universidade Academia de Humanismo Cristiano, 2007). Pesquisador do Centro de Investigación en Educación (CIE) da Universidade Bernardo O’Higgins. Suas linhas de pesquisa são: Historia da Educação; Políticas Educativas; Formação Docente; Ensino de Historia.

E-mail: felipe.zurita@ubo.cl

(ib) http://orcid.org/ 0000-0002-4136-4340 